\title{
Viral kinetic modeling and clinical trial simulation predicts disruption of respiratory disease trials by non-pharmaceutical COVID-19 interventions
}

\author{
Simon Arsène ${ }^{1}$, Claire Couty ${ }^{1,}$, Igor Faddeenkov ${ }^{1, ~ *}$, Natacha Go ${ }^{1,}$, , Solène \\ Granjeon-Noriot ${ }^{1,}$, , Daniel Smít ${ }^{1}$, Riad Kahoul ${ }^{1}$, Ben Illigens ${ }^{1,3}$, Jean-Pierre Boissel ${ }^{1}$, \\ Aude Chevalier ${ }^{2}$, Lorenz Lehr ${ }^{2}$, Christian Pasquali ${ }^{2}$, and Alexander Kulesza ${ }^{1, \dagger}$
}

\author{
${ }^{1}$ Novadiscovery SA, Lyon, France \\ ${ }^{2}$ OM Pharma, Meyrin, Switzerland \\ ${ }^{3}$ Dresden International University, Dresden, Germany \\ †corresponding author(s): Alexander Kulesza (Alexander.Kulesza@novadiscovery.com) \\ *these authors contributed equally to this work
}

12 ABSTRACT

Clinical research in infectious respiratory diseases has been profoundly affected by non-pharmaceutical interventions (NPIs) against COVID-19. On top of trial delays or even discontinuation which have been observed in all disease areas, NPIs altered transmission pattern of many seasonal respiratory viruses which followed regular patterns for decades before the pandemic. Clinical trial design based on pre-pandemic historical data therefore needs to be put in question. In this article, we show how knowledge-based mathematical modeling can be used to address this issue. We set up an epidemiological model of respiratory tract infection (RTI) sensitive to a time dependent between-host transmission rate and coupled this model to a mechanistic description of viral RTI episodes in an individual patient. By reducing the transmission rate when the lockdown was introduced in the United Kingdom in March 2020, we were able to reproduce the perturbed 2020 RTI disease burden data. Using this setup, we simulated several NPIs scenarios of various strength (none, mild, medium, strong) and conducted placebo-controlled in silico clinical trials in pediatric patients with recurrent RTIs (RRTI) quantifying annual RTI rate distributions. In interventional arms, virtual patients aged 1-5 years received the bacterial lysate OM-85 (approved in several countries for the prevention of pediatric RRTIs) through a pro-type I immunomodulation mechanism of action described by a physiologically based pharmacokinetics and pharmacodynamics approach (PBPK/PD). Our predictions showed that sample size estimates based on the ratio of RTI rates (or the post-hoc power of fixed sample size trials) are not majorly impacted under NPIs which are less severe (none, mild and medium NPIs) than a strict lockdown (strong NPI). However, NPIs show a stronger impact on metrics more relevant for assessing the clinical relevance of the effect such as absolute benefit. This dichotomy shows the risk that successful trials (even with their primary endpoints being met) still get challenged in risk benefit assessment during the review of market authorization. Furthermore, we found that a mild NPI scenario already affected the time to recruit significantly when sticking to eligibility criteria complying with historical data. In summary, our model predictions can help rationalize and forecast post-COVID-19 trial feasibility. They advocate for gauging absolute and relative benefit metrics as well as clinical relevance for assessing efficacy hypotheses in trial design and they question eligibility criteria misaligned with the actual disease burden.

\section{Introduction}

The COVID-19 pandemic and consecutive response measures to contain the spread of SARS-CoV-2 in the form of nonpharmaceutical interventions (NPIs) have not only changed people's life and health ${ }^{1}$ but also the process of developing vaccines and potential treatments ${ }^{2}$. This has led to a rapid pursue of different immunization strategies against the virus ${ }^{3,4}$, a surge of drug repurposing and the screening of new treatment candidates ${ }^{5,6}$.

Clinical development in non-COVID-19 disease areas, however, has been substantially impaired ${ }^{7}$. Due to the high number of COVID-19 cases during the pandemic in 2020, trial initiation dropped by up to $30 \%$ in the USA ${ }^{8}$. During the first wave of the pandemic, more than 1000 trials were stopped as a consequence ${ }^{9}$. Social distancing and quarantine measures have negatively affected patients participation in clinical trials. The surge in hospitalizations of COVID-19 patients also affected personnel's capacity to conduct trials ${ }^{10}$ and has led to incomplete or delayed data collection in ongoing trials with foreseeable difficulties for patient enrolment and follow-up in upcoming trials. Trialists expect that the collateral impact of COVID-19 on clinical trials will be persist for several years ${ }^{11}$, given that intermittent containment measures are possible beyond the year 
It is made available under a CC-BY-NC-ND 4.0 International license .

$2025^{12}$.

This is especially critical for trials investigating diseases of the pulmonary system. About $10 \%$ of all trials conducted in Europe in pre-COVID-19 times were on respiratory diseases ${ }^{13,14}$. Due to COVID-19 containment measures that intend to attenuate SARS-CoV-2 transmission, respiratory disease transmission is altered at the population scale, and/or there might be under-reporting of respiratory diseases to healthcare services (see a recent systematic review by Alqahtani et al. $(2021)^{15}$ ). Recent reports show that seasonal dynamics of common respiratory tract infections (RTIs) have almost vanished during the COVID-19 pandemic ${ }^{16-19}$. In England, overall fewer cases of common cold, flu, and bronchitis have been reported during the lockdown ${ }^{20}$. Hospitalization for acute bronchiolitis in children less than 1 year old saw a significant reduction, on the order of $70-90 \%$ comparing 2020 with earlier years ${ }^{21}$. For COPD (often triggered by viral infections), healthcare professionals in Europe have reported fewer cases in community and acute hospital settings $\mathrm{s}^{22,23}$ and a decline in asthma exacerbations has been reported as well ${ }^{24}$. While this decline may be regarded as a positive side-effect of the pandemic, it is only temporary and rebounding of the respiratory disease burden can happen ${ }^{25,26}$. At the same time, respiratory disease prophylaxis and international trials are strongly affected by these drastic changes because the design of clinical trials is usually conceived from pre-pandemic settings, e.g. the sample size calculation and the choice of endpoints and eligibility are based on historical interventional and observational. Therefore, clinical trial feasibility in respiratory diseases remains an open question in the medium term.

Modeling and simulation might be an approach to address the lack in representativity of historical data if forecasts of disease transmission can be joined with clinical trial simulation. For example, simulated clinical trials have provided the means to test a multitude of design choices ${ }^{27-30}$ and became a field gaining attraction throughout regulatory agencies ${ }^{31}$. The COVID-19 pandemic has already transformed the modeling and simulation community. For example, governments rely on mathematical - often epidemiological - between-host viral transmission models to predict the evolution of the pandemic and to take evidence-based decisions ${ }^{32}$. On the other hand, viral kinetic modeling, focusing on the patient immunology and viral infection resolution, can be used to accelerate drug development ${ }^{33}$.

However, to our knowledge, there is currently no available modeling approach that can simulate RTI prophylaxis trials under COVID-19 pandemic conditions and that could serve to better inform respiratory disease trials. Based on the applicability of viral kinetic models on the population and individual scale (i.e., immunology) for a broad variety of viruses, we hypothesized that a knowledge-based mechanistic model as schematized in Figure 1 could be used for in silico RTI prophylaxis trial simulation and to forecast trial feasibility. After matching known viral disease burden seasonality, intra- and inter-patient variability in RTI resolution and efficacy data, we built a mechanistic model and simulated placebo-controlled in silico trials in 1-5 year old pediatric patients with recurrent RTIs (RRTI) treated with the immunomodulator OM-85 (an agent approved in several countries for RTI prophylaxis in this population used here as a case study) under 4 different hypotheses of NPI intensities and assessed efficacy and benefit metrics as a function of NPI intensity. Subsequently, we explored sample size and recruitment considerations as aspects of trial feasibility.

\section{Results}

\section{Effect of NPIs on RTI disease burden}

Our epidemiological model is based on a compartmental approach describing susceptible, infected, recovered and again susceptible (SIRS) individuals and explicitly describes transmission, recovery, and immunity loss rates (Figure 2a, Methods). As representative comparator, we used the 5-year average and the 2019-2020 upper and lower RTI (URTI and LRTI) incidence from the communicable and respiratory disease report 2019 to 2020 published in the UK by the Royal College of General Practitioners (RCGP) ${ }^{34}$ (points and full lines in Figure 2b). To model NPI, starting at week 12 in 2020, we decreased the scaling factor of the viral transmission rate $\left(b_{0}\right.$, Supplementary Methods: Between-host SIRS model) by $17.5 \%$ to reproduce the difference between the unperturbed 5-year average and the perturbed 2019-2020 URTI and LRTI incidence with lockdown. Results of the simulations are displayed as dashed lines in Figure 2b. Simulations and data show a similar strong decline of the disease incidence with the beginning of the lockdown in the UK during March 2020 (week 10-14) ${ }^{35}$ while the 2019-2020 disease burden closely follows the 5-year average (as reported in Lacobucci et al. $(2020)^{20}$ ). With the adjusted transmission rate and otherwise unchanged parameters, the root mean square deviation (RMSD) for the weekly incidence per 100,000 of the simulation vs. data are 82 and 96 (unperturbed simulation vs. 5-year average data and perturbed simulation vs. 2019-2020, data, respectively), which is smaller than the variability within the observed data before lockdown (RMSD of 102 for the 5-year average vs. 2019-2020 data for the time points considered). Furthermore, reproduction of RTI incidence broken down into URTIs and LRTIs (Supplementary Figure S1) shows convincing capability to describe the effect of transmission perturbation on RTIs. Supported by this agreement, we applied this epidemiological model to modulate the instantaneous probability of exposure to RTI-causing viruses in our in silico trials with four different NPI scenarios. 


\section{Effect of NPIs on efficacy of RTI prophylaxis}

To represent the effect of different NPI scenarios for a 2-year clinical trial (when NPIs is introduced at the end of month 12, as an illustrative example), we defined scenarios where the transmission rate is decreased by $5 \%, 15 \%$ and $25 \%$ (Figure $3 \mathrm{a}$ ). We quantified the effect of NPIs on the efficacy of RTI prophylaxis by running in silico clinical trials using our mechanistic model applied to the oral immunomodulator OM-85 in a pediatric population suffering from recurrent RTIs. For assessing the efficacy of a prophylactic treatment absolute and relative metrics have been suggested ${ }^{36,37}$ and therefore we report model predictions for these metrics (Figure 3, Methods): absolute benefit (AB, difference between rate of RTI in both groups, Figure 3b), event rate ratio (ERR, ratio of RTI rates between both groups, Figure 3c), and two-dimensional analysis of rates of RTIs in treated vs. untreated patients (Effect Model, Methods: Efficacy analysis, Figure 3d). We define here the RTI rate as the number of RTIs counted during the 12-month follow-up period (year 2 of the trial).

The absolute benefit of OM-85 decreases in parallel to the reduction of the transmission rate which leads to a decrease in the number of preventable episodes: no reduction of the transmission rate: $1.53-2.74,5 \%$ reduction: $1.16-1.98,15 \%$ reduction: 0.60-1.14 and 25\% reduction: 0.08-0.46 prevented RTI episodes. Assuming that an AB of 1 prevented RTI episode per year would be clinically relevant in a given context (see Discussion), only NPI-induced transmission rate reduction $<15 \%$ fulfills this criterion.

The ERR quantifies efficacy based on event rates in the treated group relative to the control group. It is a common metric for performing statistical hypothesis testing with negative binomially distributed count data and may also be used for sample size estimations. We find that the ERR does not vary considerably in all but the strongest NPI scenario (no reduction of the transmission rate: $0.50-0.69,5 \%$ reduction: $0.53-0.70,15 \%$ reduction: $0.51-0.73$ and $25 \%$ reduction: $0.50-0.87$, Figure $3 \mathrm{c}$ ). In consequence, all analyses based on the ERR (i.e., sample size estimations or post-hoc power analyses) are expected to be only affected under strong NPI (e.g. strict lockdown).

To harmonize the interpretation of the different efficacy metrics (see e.g., Tripepi et al. ${ }^{36}$ ), we compared the RTI rates in the treated group (Rt) vs. RTI rates in the control group (Rc) directly in a two-dimensional analysis (Effect Model, 3d). Because $\mathrm{Rc}$ is often used to define the risk for RTI, this analysis characterizes the efficacy as a function of the risk. In all scenarios with nonzero NPI-induced viral transmission rate reduction in year 2, virtual patients experienced fewer RTIs than in year 1 ( $\geq 5$; required by eligibility criterion, no lockdown in year 1). A transmission rate reduction by $5 \%$ showed a reduction of 1.1 RTIs on average (control group RTI rates are 4.0 vs 5.1 with $5 \%$ reduction, p<.001, two-tailed Students t-test). Transmission rate reduction by $15 \%$ and $25 \%$ showed reduction of RTI rates of 2.8 and 4.3 RTIs with respect to the non-perturbed scenario (control group RTI rate are 2.3 and 0.8 vs. 5.1 with $0 \%$ reduction, both $\mathrm{p}$ values are <.001).

We then re-analyzed the efficacy distributions after the 12-month follow-up during the perturbed year 2 in relation to thresholds or for assumed clinical relevance $(\mathrm{AB}$ and $\mathrm{Rc}$ ) and statistical significance of the trial (ERR) directly in the $\mathrm{x}-\mathrm{y}$ plane of Figure 3d. We indicate a region matching three conditions (orange area in Figure 3d, lower right quadrant): i) recurrent RTI with more than 3 RTIs per year (in real-life clinical trials recurrent RTI is often defined as 3 or more RTIs in the previous year and clinical benefit is considered to prevent recurrence of RTI) as well as ii) an absolute benefit of at least 1 RTI per year under which significant clinical benefit becomes less evident to demonstrate and iii) a rate reduction of $20 \%$ in RTI rate which is a typical hypothesis for confirmatory trial design efficacy (that can be demonstrated with reasonable sample size and be clinically relevant). The percentage of in silico clinical trials complying with all three criteria is $92.8 \%, 0.0 \%$ and $0.0 \%$ for the mild, medium, and strong NPI scenarios, respectively compared to $98.2 \%$ when no NPI is applied. We thus regard trials conducted as feasible when viral transmission rates are reduced by $5 \%$ but not more than $15 \%$, even though they may still meet their endpoints (given that patient selection is not impaired in our simulation scenario, see Figure $3 \mathrm{a}$ ).

\section{Effect of NPI scenario on recruitment}

We gauged recruitment issues for RTI prophylaxis trials with estimations of the sample size estimated for a hypothesized efficacy in a given at-risk population (as a function of NPI strength) and needed power along with a more practical time-to-recruit consideration for given eligibility criteria (Figure 3e-f).

The sample size estimations commonly used in RTI prophylaxis trial designs are based on ERR assuming that RTI count data are negative binomially distributed. We have therefore used a sample size estimation algorithm (Methods) using the ERR (and negative binomial dispersion coefficient) obtained from Rc and Rt distributions in our in silico trials for a significance level of $\alpha=0.025$. Our sample size estimations as a function of NPI strength closely follow the trend of the ERR itself (no reduction of the transmission rate: $40-79,5 \%$ reduction: $42-71,15 \%$ reduction: $55-89$ and $25 \%$ reduction: $129-417$, Figure $3 \mathrm{e}$ ). Except for strong NPI, those estimates are in line with the unperturbed scenario (NPI do not affect patient selection in this example).

We estimated the time required to recruit the estimated sample sizes (Figure 3e) if NPIs were started at the beginning of year 1 (selection year) and by assuming a constant hypothetical screening rate of 1000 patients per year. A slight reduction of the transmission rate - as small as $5 \%$ - increases the time to recruit by about $50 \%$ from $0.23-0.45$ years to $0.41-0.69$ years). The medium and strongest NPI scenarios (15\% and $25 \%$ transmission rate reduction, respectively) lead to infeasible recruitment 
It is made available under a CC-BY-NC-ND 4.0 International license .

times (3-4 years and 169-546 years respectively).

\section{Discussion}

SIRS models are compartmental models which can describe infection prevalence dynamics on the population level without explicitly modeling each individual. They can be also used to reproduce periodic seasonality or attenuation of an epidemic, for example using time-dependent parametrization. Nonpharmaceutical interventions (NPIs) to help control the COVID-19 pandemic such as lockdown measures or obligations to wear masks have been imposed by policy makers. More generally, behavioral changes such as improved hygiene (e.g. washing hands more frequently), avoiding closed rooms and social distancing have been adopted in society. NPIs are assumed to be able to decrease the transmission of the virus between the infected and susceptible parts of the population. Applying this mechanistic rationale to the SIRS model, different NPI strengths can then be systematically mapped to different transmission-related parameters. The SIRS model's outcome represents therefore the average instantaneous probability density to contract a RTI in the described population under different NPI strength hypotheses. For assessing the capabilities to reproduce realistic RTI disease burden under NPI, we ran our epidemiological SIRS model (Figure 2a) with and without scaling of the viral transmission rate to replicate the (2014-2019) average 5-year URTI and LRTI disease burden in the UK versus the 2019-2020 data which captured the effect of the first lockdown through (Figure 2b). Notice that the vast majority of RTI are considered to be of viral origin ${ }^{38}$ ). We calibrated the SIRS model with heterogeneous viral prevalence data in a knowledge driven manner, which limits the degrees of freedom to calibrate the model to the data. Nevertheless, we achieved an acceptable agreement (RMSD prediction-data smaller than the 5-year average vs. 2019-2020 RMSD of 102 per 100,000 in our reference data). This agreement convinced us to use the simulated instantaneous RTI prevalence as modulator for the time-dependent instantaneous probability of an individuals exposure to viruses, where each exposure is evaluated by the within-host immunological model of RTI in an individual patient. While the use of retrospective epidemiological data about incidence rates in the time period of interest may be directly used for the coupling to the within-host model, explicit simulation allows to plausibly adjust and extrapolate this information (e.g. to another NPI scenario). As a limitation of the chosen approach, feedback from the patient scale back to the population scale (e.g. how immunomodulation can reduce viral shedding and thus transmission) is more challenging to implement. Additionally, no data are available to calibrate the OM- 85 effect on viral shedding or the efficacy under lockdown. Therefore, we needed to assume that both treatment effect and transmission are independent factors.

The central aspect of this work is to determine, rationalize and interpret the possible changes induced by lockdown and other NPIs for pandemic containment on respiratory disease trials with emphasis on RTI prophylaxis. A clinical trial has two general objectives: first, to demonstrate non-zero efficacy of the interventional strategy, a binary question with a binary answer given by a statistical test; second, to estimate the size of the clinical benefit for benefit-risk assessment. Well-designed trials fulfil both objectives through characterizing the efficacy with a quantitative measure. Not always, however, are common efficacy measures equally suitable for statistical testing and estimation of the clinical effect size. In recurrent RTIs, the event rates ratio (ERR) is often used for statistical hypothesis testing as this measure applies to negative binomially distributed count data $^{39,40}$. Nevertheless, a measured treatment efficacy that is relative to the control group event rate, is at-risk of incompletely representing the clinical benefit, in case of low event rate - as in times of NPIs to mitigate the COVID-19 pandemic. We therefore ran in silico clinical trials (based on the SIRS model, the within-host immunological model of RTI in an individual patient, virtual population and a simulation protocol resembling pediatric OM-85 trials) reproducing existing clinical efficacy data of OM-85 in a pediatric population suffering from recurrent RTI. To balance the interpretation for statistical significance versus clinical benefit considerations of these in silico trials we applied different efficacy metrics (AB and ERR) and reconciled them in a two-dimensional analysis of treated vs. untreated rates (termed Effect Model, see Methods).

Sample size estimation is of crucial importance for planning clinical trials. For this, hypotheses on expected efficacy and chosen statistical power to detect it are needed and these may have to be adapted to the current pandemic context. Second, it is important to consider how much the efficacy in a trial can differ from an efficacy hypothesis used for the planning, especially when perturbations arise after the trial has been planned or when sample size estimates based on historical data need to be used. Here, the post-hoc power obtained from the statistical analysis of the trial outcome might be perturbed under NPI. The analysis of the NPI-dependent efficacy of OM-85 for RTI prophylaxis revealed that the ERR remains unchanged over a broad range of NPI scenarios. Because ERR is used for statistical testing and sample size calculations, both the estimated sample size and the post-hoc power, are not substantially affected unless strong NPIs, such as strict lockdown, are applied. In such case, however, the post-hoc power of trials may be reduced for a given sample size and consequently trialists should consider an adapted efficacy scenario for obtaining more realistic estimates.

The situation is different for metrics of the clinical benefit which assess the benefit-risk ratio. Depending on the exact context and affected population, the definition of clinical relevance may vary. For example, prophylaxis of few LRTI episodes in neonates (often associated with inception of asthma) will be clinically relevant compared to prophylaxis of a much higher number of URTIs needed for clinical relevance in pre-school children, reflecting the different effect on patients lives and/or 
long-term consequences. First, children frequently suffer from RTIs (especially URTIs) and 3 RTI episodes per year can be considered a normal physiological behavior ${ }^{41}$. Thus, prevention of recurrence $(>3)$ of RTIs (of which most are URTIs) appears to be clinically relevant, however, our analysis has shown that under medium and strong NPI, the annual control group RTI rate is already smaller than 3, even though it was fulfilling the definition of recurrence in the unperturbed year of patient enrollment. Second, there might be a threshold for the number of prevented events for an individual (or at the population scale) which becomes relevant from a clinical or health economic standpoint. One may assume that e.g., one prevented URTI could be regarded as relevant, but we could not identify any guidance on that topic. Here again, we found that trials under medium and strong NPI scenarios do not fulfil our criterion of $\mathrm{AB}>1$ prevented RTI that could be indicative of a true clinical benefit.

The Effect Model methodology ${ }^{42,43}$, which may be obtained from meta-analyzing existing clinical data or simulation, is a tool to rationalize control vs. treated group event rates directly. Consequently both clinically meaningful and statistically demonstrable efficacy can be indicated in one analysis. In the optimal setting, the metrics used to demonstrate the efficacy with a statistical test go hand in hand with the size of the effect relevant for the benefit-risk assessment. This predictivity, however, seems to be weak under pandemic conditions given the ascertained dichotomy of NPI on AB and ERR. Under the medium NPI scenario, a substantial portion of trials with positive primary endpoint evaluation could be challenged for clinical relevance of the results and, in fact, clinical benefit-related metrics seem to be the most restrictive criteria when used to assess trial feasibility a priori. We concluded from this analysis that clinical studies need to anticipate potentially weak representativity of traditional or practical endpoints for benefit-risk assessment and that either more relevant endpoints need to be chosen or feasibility studies (including computational studies such as trial simulation) should be conducted for potential trial design adjustments.

Our simulation setup for this analysis (year 1: patient selection, year 2: treatment and follow-up period) reflects RTI prophylaxis trials whose conduction takes place during the current pandemic. Therefore, we concluded that the benefitrisk assessment of these trials should account for the currently reduced disease burden, and that supporting data (such as observational studies and models) should be used to demonstrate that a low number of prevented episodes under pandemic conditions does not necessarily mean that under normal conditions equally few episodes will be prevented.

Recruitment issues are probably the earliest and a very important indicator for difficulties to conduct clinical trials in the current COVID-19 pandemic era. For respiratory disease trials, such issues may be notably due to large sample size estimates and fewer eligible patients. NPI introduced during the follow-up period, but not during the observation period, merely scales the number of prevented events in year 2 for an already recruited population (NPI not present in year 1). Therefore, the included at-risk population (nor their immunological characteristics) are not altered in such scenarios as compared to the non-perturbed one. As the ERR used for statistical efficacy testing is a metric relative to the rate of events in the control group, it is robust towards fluctuations in the overall disease burden by design. Therefore, our analysis of estimated sample size for NPI-corrected efficacy (based on event rate ratios) did not show considerably increased recruitment needs (Figure 3e). Assuming that (e.g. for a trial with a fixed budget) an enrollment of 200 eligible patients is feasible, demonstration of efficacy in all but the strongest (25\% reduction) NPI scenario, being introduced at the beginning of year 2, remains possible with the sample size planned under no-NPI scenario. We thus conclude that estimated large sample sizes and the associated issues for recruiting high numbers of patients are currently not a major difficulty for trials which have started and completed enrolment before 2020.

By considering NPI during the observational period in year 1 of a 2-year trial, we can highlight the collateral effects of COVID-19 during patient recruitment which are caused by a reduction of the size of the pool of eligible patients. At-risk populations for a given age-range are included based on their history during a reference period (e.g., number of RTI episodes during the preceding 12 months) where risk for RTI is then defined as the average number of infections per average number of viral exposures (assumed to be a constant in that time period). In practice, in trials targeting patients aged 1 to 6 years with recurring RTI, patients with at least four to six RTI are included while the general populations suffer from e.g., only three episodes on average during the same time. This way of enriching the population with individuals at elevated risk, however, depends of the assumption that the virus exposure is a constant and that consequently the number of RTIs in the general population is also a constant. A reduction of the overall disease burden (e.g. by NPI) however, decreases the number of exposures and average number of RTIs in general. Consequently, in our simulations, small reductions of viral transmission already led to a reduced number of virtual patients who comply with any fixed definition of recurrent RTIs. We could translate this effect into a metric for recruitment difficulties, by considering the eligible fraction of the virtual population compared to the general virtual population and a defined fixed screening rate (Figure 3f). Under mild NPIs, recruitment time already increased by approximately $50 \%$, which questions the feasibility to recruit enough patients in time especially for trials with a total planned duration of 6 to 12 months. Estimated recruitment times of 3-4 years for a medium NPI scenario significantly exceed the 12 months follow-up time of most trials and can thus be considered infeasible. As these analyses do not reflect any further behavioral changes and psychological effects (e.g. fear to contract COVID-19) contributing to barriers to participate in clinical trials, the presented analysis represents an optimistic scenario. Further, we did not yet account for year-to-year fluctuations in the transmission of respiratory viruses that could add to the perturbation of NPIs (or cancel it out). Nevertheless, as it is the only scenario where recruitment time does not exceed a 12-months follow-up, the mild NPI scenario is probably the only reasonable 
It is made available under a CC-BY-NC-ND 4.0 International license .

condition compatible with recruiting enough patients for RTI prophylaxis trials under real-world conditions. Considering that during the first UK lockdown, transmission reduction by $17.5 \%$ best reproduces the disease burden data, $5 \%$ reduction as in the mild NPI scenario is a plausible assumption for a long-term effect on viral transmission (e.g., masks, a threshold number of people in events, hand sanitizers in public places). To conclude, the selection of patients with recurrent RTI based on pre-pandemic historical data would only include a very small fraction of patients, thus, we suggest considering eligibility criteria tailored to the current incidence of RTI at a given time to avoid misalignment of targeted and included population. But then, selecting the right at-risk population could become more challenging in turn.

Overall, we present here a mechanistic in silico clinical trial approach in RTI prophylaxis which can incorporate available disease burden data to output efficacy metrics relevant for assessing clinical benefits and estimating sample sizes in perturbed scenarios (or evaluating impact on the post-hoc power of a trial for a given sample size) as well as recruitment times. Mechanistic description of the transmission of respiratory viruses can thereby translate lockdown and social distancing measures into a decreased rate of RTI events in patients, and into a shift of the risk-dependent efficacy for OM-85 treatment in clinical trial simulations. We highlighted that statistical significance of efficacy may be less predictive of the clinical benefit because there are fewer events to prevent (due to collateral impact of COVID-19 containment), and consequently benefit-risk assessment based on current RTI-prophylaxis trials might be difficult to establish. Recruitment of patients can be impeded as long as intermittent lockdown or perturbations of seasonal virus transmission persist - in particular when trialists rely on pre-pandemic historical data for trial design. Several open questions remain: What are the additional adjustments required for trial design to account for the effect of the pandemic? How does the altered and shifted seasonality of respiratory viruses affect follow-up duration? Is there a potential benefit of using inclusion criteria adapted to pandemic times (such as incidence matching) and do those adaptations risk to confound efficacy? What happens if the forecast of the disease burden turns out to be wrong?

The limitations of traditional clinical trial design methodology and the proof of concept established in this Modeling \& Simulation study advocates for the use of mechanistic computational models to address these questions in detail and to support go/no-go decisions in clinical development for a wide range of RTI prophylaxis-oriented treatments. Furthermore, when trials are deemed infeasible, the models could harness RTI disease burden monitoring (and prediction of COVID-19 and its associated containment measures) to indicate the time point when delayed or stopped trials can be restarted.

\section{Methods}

\section{Modeling approach}

The in silico clinical trials in this work are simulations performed with system models using ordinary differential equations (ODEs) embedded in a virtual population approach where parameters are described by statistical distributions rather than scalar values, in order to represent different sources of variability. Each virtual patient corresponds to a vector of parameter values, drawn from the corresponding statistical distribution. Similar to a real clinical trial protocol, an in silico study protocol defines the use of the model, virtual population, simulation scenarios and statistical analyses to answer a question of interest.

\section{Multiscale RTI disease and treatment model}

The core element of the computational approach is the coupling of a within-host mechanistic disease model, representing the viral and immune dynamics, with a between-host disease burden model, representing the viral dynamics at the population-scale with a Susceptible, Infectious, Recovered, Susceptible (SIRS) framework, to obtain a multiscale RTI and immunomodulation model (Figure 1). The immunological and the SIRS models are both ODEs-based deterministic models (equations and parameters provided in the Supplementary Methods).

\section{Immunological within-host viral infection model}

The immunological model, implementing lytic versus nonlytic immune mechanisms during viral infection, was designed based on Wodarz et al. (2002) $)^{44}$ to simulate the within-host dynamics in response to respiratory virus exposure (co-infections are not accounted) (bottom part of Figure 1, Supplementary Methods, Supplementary Figure S2, Supplementary Table S1). To translate individual occurrences of RTI events for a given patient over time into the distributions of RTI rates in the population, inter- and intra-individual variability need to be taken into account. For this, stochastic processes determine time points of viral exposure and current state of antiviral defenses. A patient-specific state of antiviral defenses (immuno-competence) is therefore distributed in the population and a layer of random fluctuations is added around each individual value. Both distributions were calibrated so that the RTI distribution in the virtual population represents a reference RTI prevalence distribution data set (obtained from a reference birth cohort ${ }^{41}$ ). Describing age as a covariate for this distribution required inclusion of a maturation term into the immune effector functions to reproduce the higher risk for RTI in young children due the still developing immune system (Supplementary Methods, Supplementary Figure S3).

Between-host viral infection and disease burden model

RTI disease burden was simulated using a SIRS model (described in Supplementary Methods: Between-host SIRS model) 
It is made available under a CC-BY-NC-ND 4.0 International license .

inspired by general literature on such models ${ }^{45}$. This SIRS model accounts for the seasonality of infection in an averaged manner in a given population; it is based on time-dependent transmission rates of selected viruses reproducing the seasonality of upper and lower RTIs attributed to RSV, RV and Influenza viruses (Supplementary Figure S4, Supplementary Table S2). We first ran the epidemiological model alone with NPI-adjusted transmission rate (reduction of mean transmission rates $b_{0}$ by $0 \%$, 5\%, 15\%, 25\%) and compared it with data digitized from the communicable and respiratory disease reports from 2019 to 2020 published in the UK by the Royal College of General Practitioners (RCGP) ${ }^{34}$. The outcome of the SIRS model was then used to provide the data for the time-dependent instantaneous prevalence of RTI for the rest of the model.

\section{Treatment model}

To describe the immuno-modulating effect of OM-85 in RTI prophylaxis, a physiologically based pharmacokinetics and pharmacodynamics (PBPK/PD) model is linked to the immunological model through ingress in the respiratory tract of reprogrammed type-1 innate memory like cells ${ }^{46}$, regulatory T-cells ${ }^{47-49}$, and polyclonal IgA producing plasma cells $s^{50,51}$ originating from the intestinal Peyer's patches (Supplementary Figure S5) according to the current understanding of OM-85's mechanism of action. Implementation of administration, distribution, metabolism and excretion follows common published approaches (Supplementary Methods: PBPK/PD model of OM-85 effect). In absence of OM-85 PK data, the unknown PBPK drug-specific parameters were calibrated using rodent PK data of a similar product (OM-89) ${ }^{52,53}$ and were allometrically scaled to human physiology (Supplementary Figure S6, Supplementary Table S3). Unknown PD-relevant parameters were calibrated and checked using two sets of human PD response data under different treatment regimens (Supplementary Figure S7-8, Supplementary Table S4). Calibration of remaining parameters that quantify the size of the efficacy of OM- 85 was performed based on the meta-analysis of Yin et al. ${ }^{54}$ (Supplementary Methods: Calibration of OM-85 clinical efficacy, Supplementary Figure S10-11).

\section{In silico clinical trial simulations}

We simulated placebo-controlled parallel two-arm trials of RTI prevention with OM-85 in pediatric subjects with 24 months duration (observational period of 1 year followed by a follow-up period of 1 year composed of 3 consecutive months of treatment followed by 9 without any treatment). A virtual population of more than 100,000 virtual patients was generated. The entire virtual population was screened during the observational period in the first year of the trial. After the first year, eligibility criteria were evaluated and randomization was performed. In line with the range of annual RTI episodes typically defining recurrent RTIs (3-6), children that experienced at least 5 RTIs were included into the follow-up period. Included virtual subjects were randomly allocated with equal weight to the interventional and control arms. During the first 3 months of the follow-up period, OM-85 was administered every day during the first 10 consecutive days of each month, in line with the current approved dosing regimen of OM-85 in the prevention of RTIs. The primary outcome was the number of RTIs during one-year follow-up, which was assessed at the end of trial. 600 in silico clinical trials were simulated for each of the four different NPI scenarios (reduction of the transmission rate by $0,5 \%, 15 \%, 25 \%$ ) by randomly sampling 50 subjects per arm from the screened virtual population (studies meta analyzed by Yin et al. ${ }^{54}$ have enrolled in average 45.4 patients per arm).

\section{Mechanistic uncertainty analysis}

To assess the effect of main drivers of uncertainty represented in the model (i.e., treatment efficacy related parameters) we simulated different mechanistic scenarios in parallel throughout the modeling procedure and analyses. We used 12 different conditions (testing different immunogenic hypotheses on the effect of OM-85, (Supplementary Methods:Mechanistic uncertainty management) for each of the 4 NPI scenarios. We pooled the results, as these variations of OM-85 immunogenicity may indeed contribute to the overall outcome (e.g. efficacy) variability.

\section{Efficacy analysis}

The Effect Model approach ${ }^{42,55}$ is a tool which relates the rates (or risks) of events without treatment (Rc) and with (Rt), as supported by empirical evidence, simulations and theoretical considerations ${ }^{56-59}$. While simulations can be conducted for the same patient in different arms in in silico trials and yield paired observations, the Effect Model can also be reconciled with meta-analyses ${ }^{56,60}$. Here we have used a similar approach which compares RTI rates in a series of individual in silico clinical trials, thus not reporting individual, but risk-stratified group metrics. As efficacy metrics, we consider absolute benefit (AB) and the event rate ratio (ERR). Average ERR and $\mathrm{AB}$ were assessed at one-year follow up. AB determined from a single in silico trial is the arithmetic difference between mean RTI rate in the control group (Rc) and in the treatment group (Rt). ERR refers to the ratio Rt/Rc. Distribution of the ERR and AB per scenario contain pooled results for different mechanistic conditions and visualized as maximum interquartile range (defined as the difference between maximum $75^{\text {th }}$ percentile and minimum $25^{\text {th }}$ percentile across mechanistic conditions). AB and ERR were analyzed with a paired t-test/ANOVA with $\alpha$ level set at .05 . 


\section{Sample size and recruitment estimation}

Sample size calculations for primary endpoint analyses of RTI prophylaxis trials require an adapted statistical method for overdispersed count data. We performed generalized linear regression analysis with negative binomial distributions (mean and dispersion parameter, $g \mathrm{~lm}$ function of the R package MASS) for the subsequent use of these parameters in the sample size calculation method proposed by Zhu et al. (2013) ${ }^{61}$. Calculations employed the power.nb.test function of the MKmisc package given the ratio of rates in both trial arms, average dispersion parameter, an $\alpha$ of 0.025 and correction for average study duration (e.g. due to dropout $\kappa=0.75$ ). Based on sample size calculations and the fraction of the entire Virtual Population eligible for inclusion, time to recruitment was calculated, assuming that in a typical study in respiratory diseases a screening rate of 1000 patients per year can be achieved per center.

\section{References}

1. Tušl, M., Brauchli, R., Kerksieck, P. \& Bauer, G. F. Impact of the COVID-19 crisis on work and private life, mental well-being and self-rated health in german and swiss employees: a cross-sectional online survey. BMC Public Heal. 21, DOI: $10.1186 / \mathrm{s} 12889-021-10788-8$ (2021).

2. Ayati, N., Saiyarsarai, P. \& Nikfar, S. Short and long term impacts of COVID-19 on the pharmaceutical sector. DARU J. Pharm. Sci. 28, 799-805, DOI: 10.1007/s40199-020-00358-5 (2020).

3. Taylor, P. C. et al. Neutralizing monoclonal antibodies for treatment of COVID-19. Nat. Rev. Immunol. 21, 382-393, DOI: 10.1038/s41577-021-00542-x (2021).

4. Kyriakidis, N. C., López-Cortés, A., González, E. V., Grimaldos, A. B. \& Prado, E. O. SARS-CoV-2 vaccines strategies: a comprehensive review of phase 3 candidates. npj Vaccines 6, DOI: 10.1038/s41541-021-00292-w (2021).

5. Heimfarth, L., Serafini, M. R., Martins-Filho, P. R., de Souza Siqueira Quintans, J. \& Quintans-Júnior, L. J. Drug repurposing and cytokine management in response to COVID-19: A review. Int. Immunopharmacol. 88, 106947, DOI: 10.1016/j.intimp.2020.106947 (2020).

6. Gysi, D. M. et al. Network medicine framework for identifying drug-repurposing opportunities for COVID-19. Proc. Natl. Acad. Sci. 118, e2025581118, DOI: 10.1073/pnas.2025581118 (2021).

7. Sathian, B. et al. Impact of COVID-19 on clinical trials and clinical research: A systematic review. Nepal J. Epidemiol. 10, 878-887, DOI: 10.3126/nje.v10i3.31622 (2020).

8. Unger, J. M. \& Xiao, H. The COVID-19 pandemic and new clinical trial activations. Trials 22, DOI: 10.1186/ s13063-021-05219-3 (2021).

9. Upadhaya, S., Yu, J. X., Hodge, J. \& Campbell, J. COVID-19 impact on oncology clinical trials: a 1-year analysis. Nat. Rev. Drug Discov. 20, 415-415, DOI: 10.1038/d41573-021-00086-8 (2021).

10. Upadhaya, S. et al. Impact of COVID-19 on oncology clinical trials. Nat. Rev. Drug Discov. 19, 376-377, DOI: 10.1038/d41573-020-00093-1 (2020).

11. Ledford, H. The COVID pandemic's lingering impact on clinical trials. Nature DOI: 10.1038/d41586-021-01569-9 (2021).

12. Kissler, S. M., Tedijanto, C., Goldstein, E., Grad, Y. H. \& Lipsitch, M. Projecting the transmission dynamics of SARS-CoV-2 through the postpandemic period. Science 368, 860-868, DOI: 10.1126/science.abb5793 (2020).

13. Ruopp, M., Chiswell, K., Thaden, J. T., Merchant, K. \& Tsalik, E. L. Respiratory tract infection clinical trials from 2007 to 2012. a systematic review of ClinicalTrials.gov. Annals Am. Thorac. Soc. 12, 1852-1863, DOI: 10.1513/annalsats. 201505-291oc (2015).

14. Bodini, R. et al. Epidemiology of clinical trials of medicines in respiratory diseases in europe and italy. Respir. Medicine 125, 8-11, DOI: 10.1016/j.rmed.2017.02.009 (2017).

15. Alqahtani, J. S. et al. Reduction in copd exacerbations during covid-19: a systematic review and meta-analysis. medRxiv DOI: $10.1101 / 2021.05 .17 .21257335$ (2021).

16. Dilworth, T. J. \& Brummitt, C. F. Reduction in ambulatory visits for acute, uncomplicated bronchitis: an unintended but welcome result of the coronavirus disease 2019 (COVID-19) pandemic. Infect. Control. \& Hosp. Epidemiol. 1-2, DOI: 10.1017/ice.2020.1233 (2020).

17. Jones, N. How COVID-19 is changing the cold and flu season. Nature 588, 388-390, DOI: 10.1038/d41586-020-03519-3 (2020).

18. Varela, F. H. et al. Absence of detection of RSV and influenza during the COVID-19 pandemic in a brazilian cohort: Likely role of lower transmission in the community. J. Glob. Heal. 11, DOI: 10.7189/jogh.11.05007 (2021). 
19. Brusselen, D. V. et al. Bronchiolitis in COVID-19 times: a nearly absent disease? Eur. J. Pediatr. 180, 1969-1973, DOI: 10.1007/s00431-021-03968-6 (2021).

20. Iacobucci, G. Covid lockdown: England sees fewer cases of colds, flu, and bronchitis. $B M J \mathrm{~m} 3182$, DOI: $10.1136 / \mathrm{bmj}$. m3182 (2020).

21. Friedrich, F. et al. Early impact of social distancing in response to coronavirus disease 2019 on hospitalizations for acute bronchiolitis in infants in brazil. Clin. Infect. Dis. 72, 2071-2075, DOI: 10.1093/cid/ciaa1458 (2020).

22. Alsallakh, M. A. et al. Impact of COVID-19 lockdown on the incidence and mortality of acute exacerbations of chronic obstructive pulmonary disease: national interrupted time series analyses for scotland and wales. BMC Medicine 19, DOI: 10.1186/s12916-021-02000-w (2021).

23. Faria, N., Costa, M. I., Gomes, J. \& Sucena, M. Reduction of severe exacerbations of COPD during COVID-19 pandemic in portugal: A protective role of face masks? COPD: J. Chronic Obstr. Pulm. Dis. 18, 226-230, DOI: $10.1080 / 15412555.2021 .1904387$ (2021).

24. Salciccioli, J. D. et al. Effect of COVID-19 on asthma exacerbation. The J. Allergy Clin. Immunol. In Pract. 9, 28962899.e1, DOI: 10.1016/j.jaip.2021.04.038 (2021).

25. Agha, R. \& Avner, J. R. Delayed seasonal rsv surge observed during the covid-19 pandemic. Pediatrics 148, DOI: 10.1542/peds.2021-052089 (2021).

26. Hussain, F., Kotecha, S. \& Edwards, M. O. Rsv bronchiolitis season 2021 has arrived, so be prepared! Arch. Dis. Child. DOI: 10.1136/archdischild-2021-322835 (2021).

27. Holford, N. H. G., Kimko, H. C., Monteleone, J. P. R. \& Peck, C. C. Simulation of clinical trials. Annu. Rev. Pharmacol. Toxicol. 40, 209-234, DOI: 10.1146/annurev.pharmtox.40.1.209 (2000).

28. Pappalardo, F., Russo, G., Tshinanu, F. M. \& Viceconti, M. In silico clinical trials: concepts and early adoptions. Briefings Bioinforma. 20, 1699-1708, DOI: 10.1093/bib/bby043 (2018).

29. Bajard, A. et al. An in silico approach helped to identify the best experimental design, population, and outcome for future randomized clinical trials. 69, 125-136, DOI: 10.1016/j.jclinepi.2015.06.024 (2016).

30. Wang, Y. et al. Model-informed drug development: Current US regulatory practice and future considerations. Clin. Pharmacol. \& Ther. 105, 899-911, DOI: 10.1002/cpt.1363 (2019).

31. Li, L. et al. CPT: pharmacometrics \& systems pharmacology 8, 59, DOI: 10.1002/psp4.12368 (2019).

32. Adam, D. Special report: The simulations driving the world's response to COVID-19. Nature 580, 316-318, DOI: 10.1038/d41586-020-01003-6 (2020).

33. Dodds, M. G., Krishna, R., Goncalves, A. \& Rayner, C. R. Model-informed drug repurposing: Viral kinetic modelling to prioritize rational drug combinations for COVID-19. Br. J. Clin. Pharmacol. 87, 3439-3450, DOI: 10.1111/bcp.14486 (2020).

34. Royal College of General Practitioners (RCGP) Research \& Surveillance Centre. RSC communicable and respiratory disease report for england - week 31. Tech. Rep., University of Oxford, University of Surrey (2020).

35. Timeline of uk coronavirus lockdowns, march 2020 to march 2021. https://www.instituteforgovernment.org.uk/sites/ default/files/timeline-lockdown-web.pdf. Accessed: 2021-07-06.

36. Tripepi, G., Jager, K., Dekker, F., Wanner, C. \& Zoccali, C. Measures of effect: relative risks, odds ratios, risk difference, and number needed to treat. Kidney international 72, 789-791, DOI: 10.1038/sj.ki.5002432 (2007).

37. Boissel, J.-P., Cogny, F., Marko, N. \& Boissel, F.-H. From clinical trial efficacy to real-life effectiveness: Why conventional metrics do not work. 6, 125-132, DOI: 10.1007/s40801-019-0159-z (2019).

38. Schaad, U. B. Prevention of paediatric respiratory tract infections: emphasis on the role of OM-85. 14, 74-77, DOI: 10.1183/09059180.05.00009506 (2005).

39. Young, L. J., Campbell, N. L. \& Capuano, G. A. Analysis of overdispersed count data from single-factor experiments: A comparative study. 4, 258, DOI: 10.2307/1400385 (1999).

40. Aban, I. B., Cutter, G. R. \& Mavinga, N. Inferences and power analysis concerning two negative binomial distributions with an application to MRI lesion counts data. 53, 820-833, DOI: 10.1016/j.csda.2008.07.034 (2009).

41. Carlsson, C. J. et al. Duration of wheezy episodes in early childhood is independent of the microbial trigger. J. Allergy Clin. Immunol. 136, 1208-1214.e5, DOI: 10.1016/j.jaci.2015.05.003 (2015). 
42. Boissel, J.-P. et al. Towards personalized medicine: exploring the consequences of the effect model-based approach. Pers. Medicine 8, 581-586, DOI: 10.2217/pme.11.54 (2011).

43. Boissel, J.-P., Auffray, C., Noble, D., Hood, L. \& Boissel, F.-H. Bridging systems medicine and patient needs. CPT: Pharmacometrics \& Syst. Pharmacol. 4, 135-145, DOI: 10.1002/psp4.26 (2015).

44. Wodarz, D. The importance of lytic and nonlytic immune responses in viral infections. Trends Immunol. 23, 194-200, DOI: $10.1016 / \mathrm{s} 1471-4906(02) 02189-0$ (2002).

45. Kermack, W. O. \& McKendrick, A. G. A contribution to the mathematical theory of epidemics. 115, 700-721, DOI: 10.1098/rspa.1927.0118 (1927).

46. Wang, Peng, H. \& Tian, Z. Innate lymphoid cell memory. Cell. \& Mol. Immunol. 16, 423-429, DOI: 10.1038/ s41423-019-0212-6 (2019).

47. Navarro, S. et al. The oral administration of bacterial extracts prevents asthma via the recruitment of regulatory $t$ cells to the airways. 4, 53-65, DOI: 10.1038/mi.2010.51 (2010).

48. Strickland, D. H. et al. Boosting airway t-regulatory cells by gastrointestinal stimulation as a strategy for asthma control. 4, 43-52, DOI: $10.1038 / \mathrm{mi} .2010 .43$ (2010).

49. Fu, R. et al. Broncho-vaxom attenuates allergic airway inflammation by restoring GSK3-related t regulatory cell insufficiency. 9, DOI: 10.1371/journal.pone.0092912 (2014).

50. Emmerich, B., Pachmann, K., Milatovic, D. \& Emslander, H. Influence of OM-85 BV on Different Humoral and Cellular Immune Defense Mechanisms of the Respiratory Tract. Respiration 59, 19-23, DOI: 10.1159/000196126 (2009).

51. Lusuardi, M. et al. Local Airways Immune Modifications Induced by Oral Bacterial Extracts in Chronic Bronchitis. Chest 103, 1783-1791, DOI: 10.1378/chest.103.6.1783 (1993).

52. van Dijk, A., Bauer, J., Sedelmeier, E. A. \& Bessler, W. G. Absorption, kinetics, antibody-bound and free serum determination of a 14C-labeled Escherichia coli extract after single oral administration in rats. Arzneimittelforschung 47, 329-334 (1997).

53. Burckhart, M. F., Mimouni, J. \& Fontanges, R. Absorption kinetics of a 14c-labelled escherichia coli extract after oral administration in mice. Arzneimittel-Forschung 47, 325-328 (1997).

54. Yin, J., Xu, B., Zeng, X. \& Shen, K. Broncho-vaxom in pediatric recurrent respiratory tract infections: A systematic review and meta-analysis. Int. immunopharmacology 54, 198-209, DOI: 10.1016/j.intimp.2017.10.032 (2018).

55. Boissel, J.-P., Kahoul, R., Marin, D. \& Boissel, F.-H. Effect model law: An approach for the implementation of personalized medicine. JPM 3, 177-190, DOI: 10.3390/jpm3030177 (2013).

56. Boissel, J.-P., Collet, J.-P., Lievre, M. \& Girard, P. An effect model for the assessment of drug benefit: example of antiarrhythmic drugs in postmyocardial infarction patients. J. cardiovascular pharmacology 22, 356-363 (1993).

57. Boissel, J.-P. Individualizing aspirin therapy for prevention of cardiovascular events. JAMA 280, 1949, DOI: 10.1001/jama. 280.22.1949 (1998).

58. Glasziou, P. P. \& Irwig, L. M. An evidence based approach to individualising treatment. BMJ 311, 1356-1359, DOI: 10.1136/bmj.311.7016.1356 (1995).

59. Wang, H., Boissel, J.-P. \& Nony, P. Revisiting the relationship between baseline risk and risk under treatment. Emerg. Themes Epidemiol. 6, DOI: 10.1186/1742-7622-6-1 (2009).

60. Boissel, J.-P. et al. New insights on the relation between untreated and treated outcomes for a given therapy effect model is not necessarily linear. J. clinical epidemiology 61, 301-307 (2008).

61. Zhu, H. \& Lakkis, H. Sample size calculation for comparing two negative binomial rates. Stat. Med. 33, 376-387, DOI: 10.1002/sim.5947 (2013).

\section{Acknowledgements}

We acknowledge feedback by Jim Bosley and Shiny Martis B. on the manuscript draft.

\section{Author contributions statement}

A.K., S.A., L.L., C.P and A.C. supervised the study. S.A, C.C, I.F., N.G., S.G.N., D.S., developed the model, performed simulations and analyzed the results. S.A. and A.K wrote the manuscript. All authors contributed to the discussion of the results and reviewed the manuscript. 
medRxiv preprint doi: https://doi.org/10.1101/2021.11.09.21266145; this version posted November 10, 2021. The copyright holder for this preprint (which was not certified by peer review) is the author/funder, who has granted medRxiv a license to display the preprint in perpetuity. It is made available under a CC-BY-NC-ND 4.0 International license .

\section{Additional information}

AK, SA, CC, IF, NG, SGN, DS, RK, BI, JPB are employees of Novadiscovery. AC, LL, CP are employees of OM Pharma. Novadiscovery and OM Pharma funded the study.

The datasets generated during and/or analysed during the current study are available from the corresponding author on 491 reasonable request. 


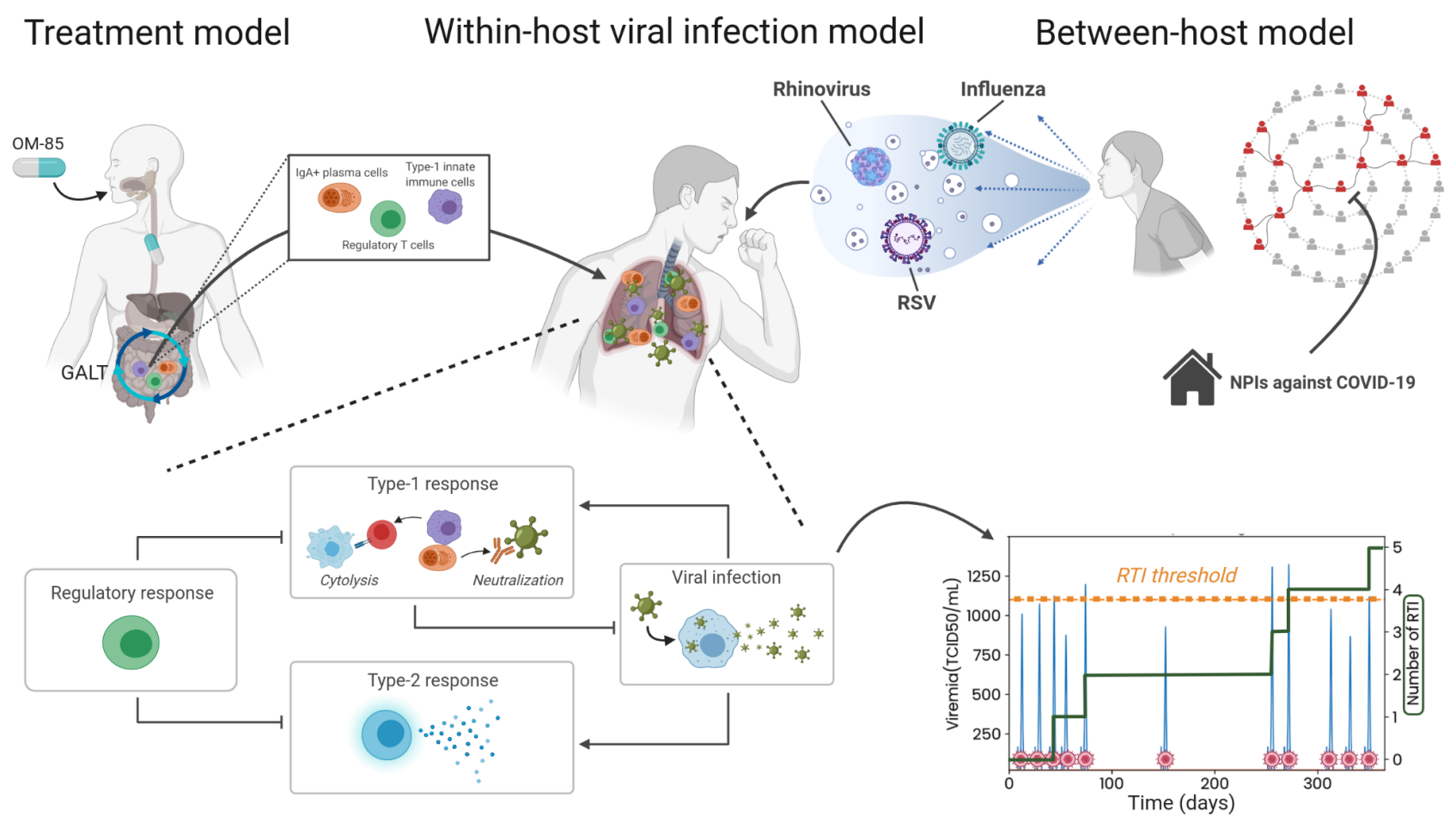

Figure 1. Multi-scale in silico approach to incorporate within-host and between-host respiratory tract infection (RTI) model as well as a treatment model with bacterial lysate OM-85. The model is used to assess feasibility of clinical trials in prophylaxis of RTIs during COVID-19 pandemic. The transmission of the major respiratory pathogens RSV, rhinovirus and influenza type A and B viruses is given by a seasonal SIRS model (between-host model). This model is interfaced to a within-host immunology model via a time-dependent instantaneous prevalence of infection triggering or not viral exposure at randomly chosen time points. Individual patients are identified by their age and an immuno-competence meta-parameter impacting the immune response from which infections are included or omitted from the cumulative number of infections depending on viremia. To prevent RTIs, virtual patients are treated with the bacterial lysate OM-85 which acts through a pro-type I immunomodulation mechanism of action and which is described by a physiologically based pharmacokinetics and pharmacodynamics approach (treatment model) with downstream effects in the immunological model. The impact of COVID-19 assiocated non-pharmaceutical interventions (NPIs) are simulated by scaling of the transmission term in the between-host part of the model. Figure created with BioRender.com. 
medRxiv preprint doi: https://doi.org/10.1101/2021.11.09.21266145; this version posted November 10, 2021. The copyright holder for this preprint (which was not certified by peer review) is the author/funder, who has granted medRxiv a license to display the preprint in perpetuity.

It is made available under a CC-BY-NC-ND 4.0 International license .

a)

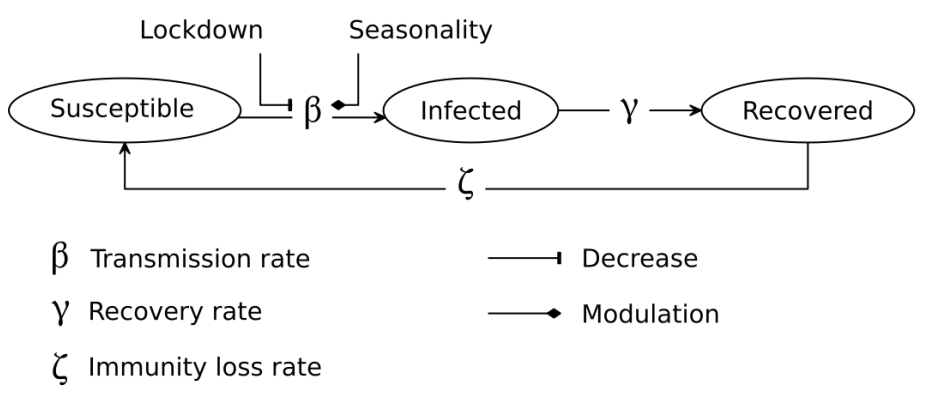

b)

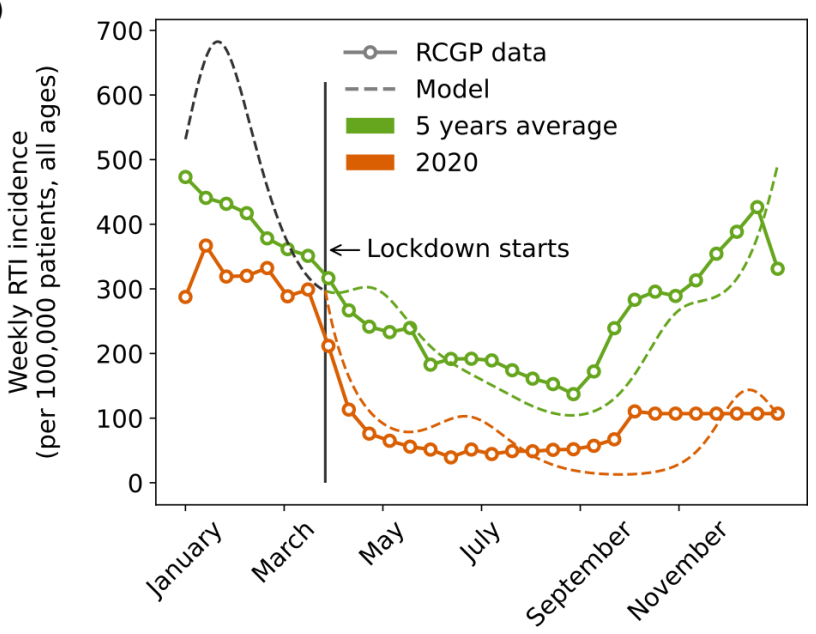

Figure 2. Between-host model based on SIRS framework allows to reproduce RTI incidence during nonpharmaceutical interventions (NPIs) to mitigate COVID-19 pandemic. a) Schematic of implemented SIRS model where NPI can be modelled by a decrease of the transmission rate. b) Comparison of model predictions (dashed lines) and data (solid lines) from RCGP $^{34}$ for RTI weekly incidence (per 100,000 all ages) for the 5 years average (green) and 2020 (orange). Lockdown was started on the $23^{\text {th }}$ of March 2020 in the UK. This date was used to implement the lockdwon in the simulations with a decrease of $17.5 \%$ of the transmission rate. 
medRxiv preprint doi: https://doi.org/10.1101/2021.11.09.21266145; this version posted November 10, 2021. The copyright holder for this preprint (which was not certified by peer review) is the author/funder, who has granted medRxiv a license to display the preprint in perpetuity.

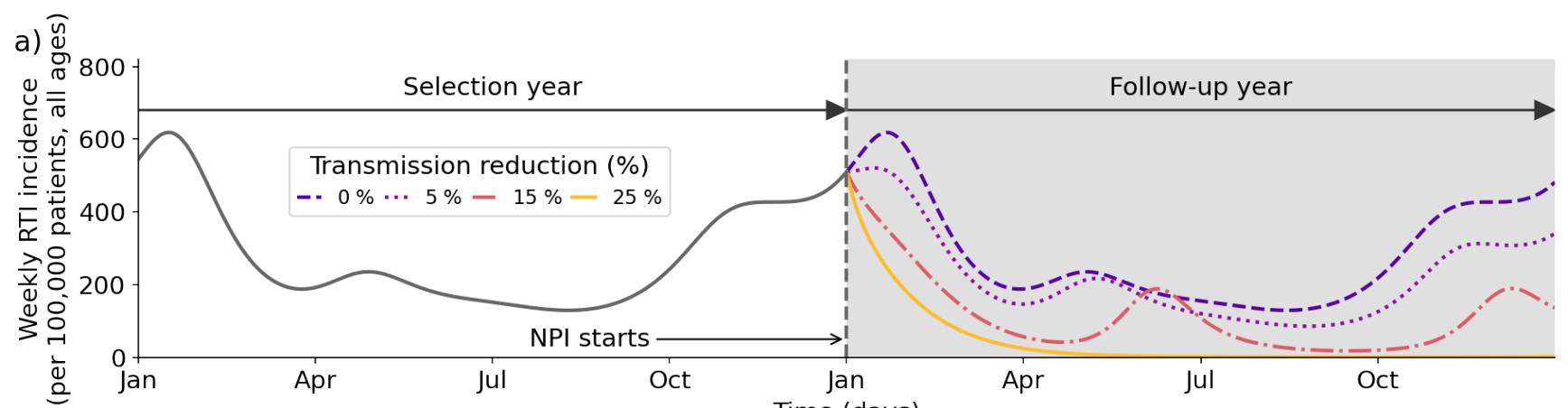

b)

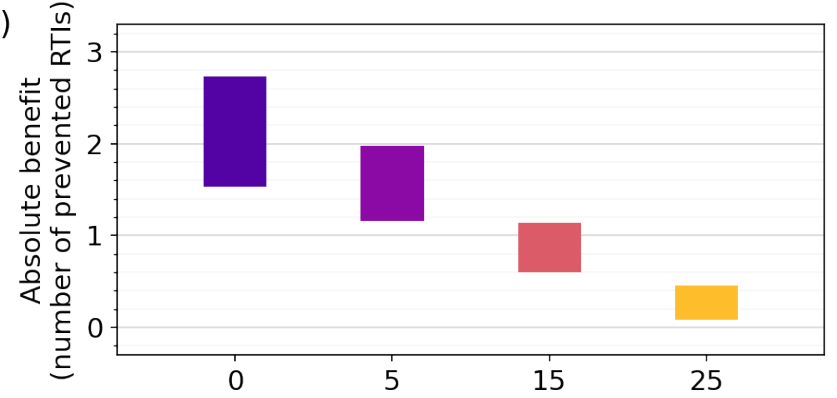

d)

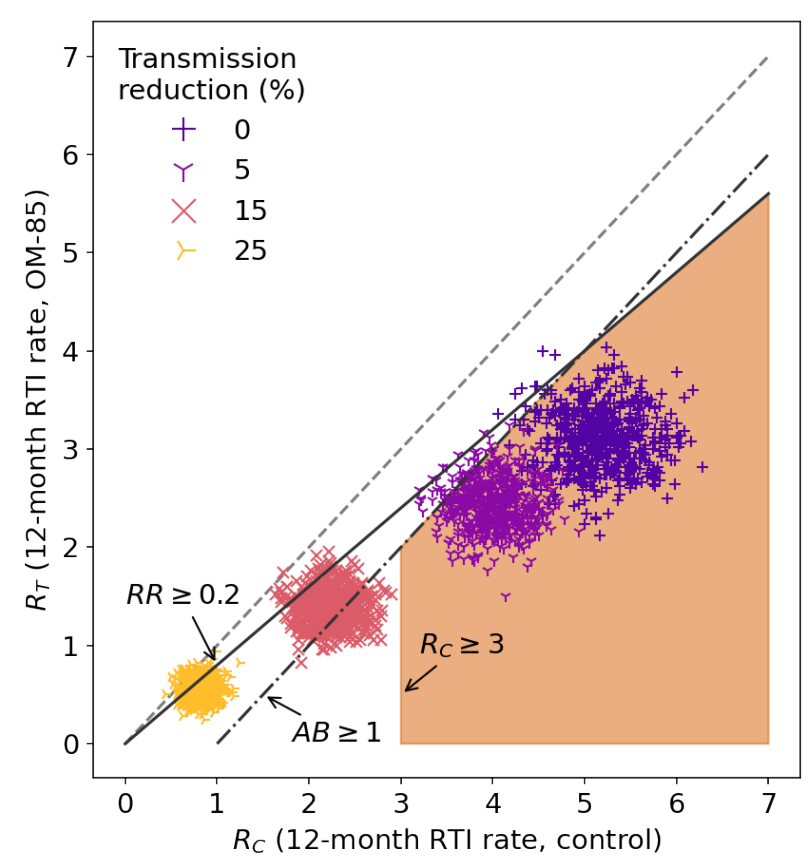

(days)

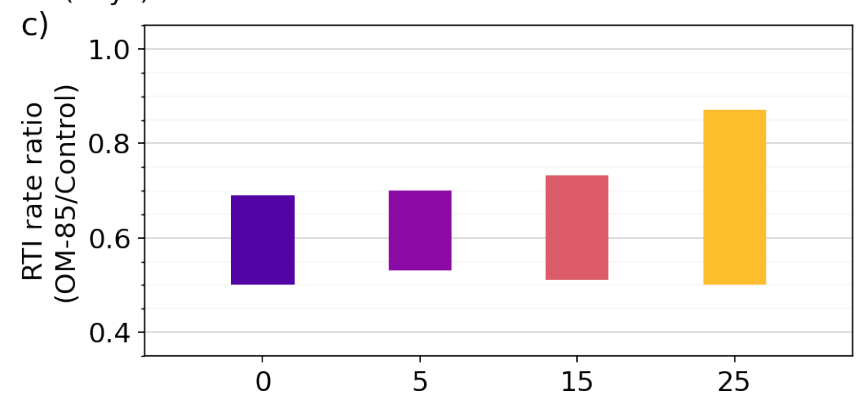

e)
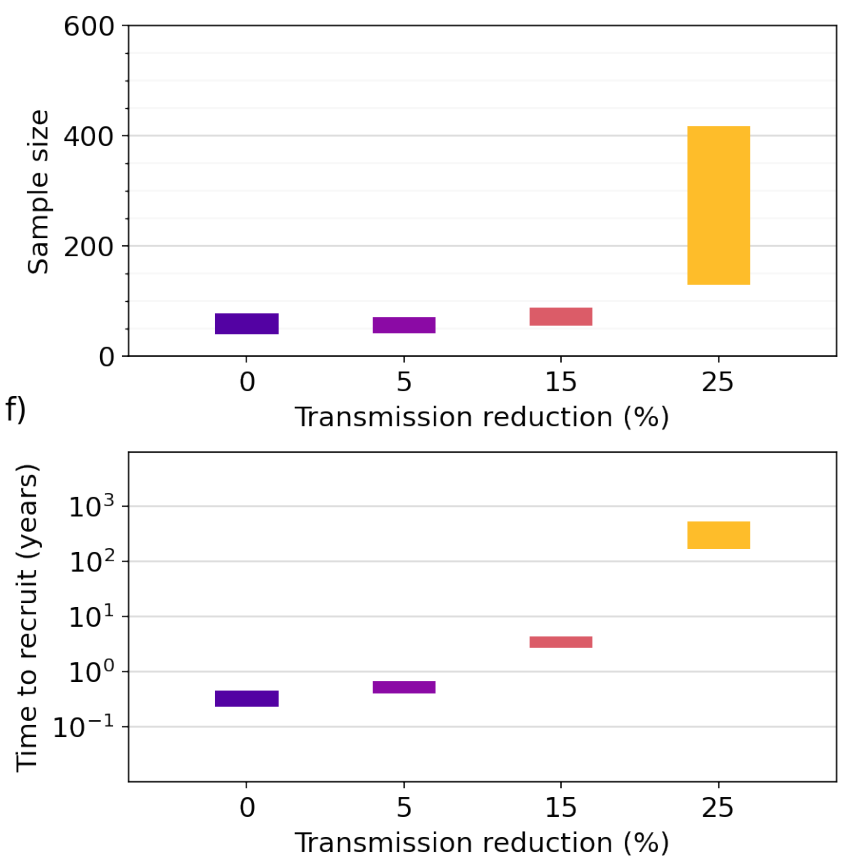
medRxiv preprint doi: https://doi.org/10.1101/2021.11.09.21266145; this version posted November 10, 2021. The copyright holder for this

preprint (which was not certified by peer review) is the author/funder, who has granted medRxiv a license to display the preprint in perpetuity.

It is made available under a CC-BY-NC-ND 4.0 International license .

Figure 3. Results of in silico clinical trials in prophylaxis of respiratory tract infections (RTIs) with four scenarios of non-pharmaceutical interventions (NPIs) against COVID-19 pandemic with increasing strength (absent, mild, medium and strong) modelled by a decrease of the transmission rate parameter (no reduction, $-\mathbf{5 \%},-\mathbf{- 1 5 \%}$ and $\mathbf{- 2 5 \%}$, respectively). For all scenarios, the simulations are run for 2 years. Year 1 is the selection year during which patients are screened and possibly included in an in silico trial. There is no NPI during year 1. The NPIs are started at the beginning of year 2 as well as the treatment (10 daily administrations of $3.5 \mathrm{mg}$ of OM- 85 from the beginning of the month for 3 consecutive months). RTIs are counted for the complete duration of year 2. a) Weekly incidence of RTIs per 100,000 is plotted for two years of simulations for the four NPI scenarios. NPI is started at the beginning of year 2. b) Distribution (maximum IQR) of absolute benefit is plotted for the four NPI scenarios. Absolute benefit can be interpreted as the number of prevented RTIs in year 2 when comparing the treated and the control group. Maximum IQR is defined as the difference between maximum $75^{\text {th }}$ percentile and minimum $25^{\text {th }}$ percentile across mechanistic conditions. c) Distribution (maximum IQR) of event RTI rate ratio (ERR, treated over control group) is plotted for the four NPI scenarios. d) Effect Model plot for the four NPI scenarios. Each in silico clinical trial is plotted (cross) with the number of RTIs in the control group as x coordinate and the number of RTIs in the treated group as y coordinate. The region of clinically relevant efficacy is indicated in orange. It is defined by at least 1 prevented RTI in absolute benefit (dashed-dotted line), at least 20\% reduction in number of RTIs (solid line) and least 3 RTIs in the control group. By that criteria, only trials with absent or mild NPI scenario are deemed feasible. e) Distribution (maximum IQR) of sample sizes per arm required to show efficacy of OM-85 treatment in reducing number of RTIs for the four NPI scenarios. f) Distribution (maximum IQR) of estimated patient screening times under the four NPI scenarios by assuming an hypothetical screening rate of 1,000 patients per year and by taking year 2 as the selection year (without treatment). 